\title{
GLOBAL FOURIER INTEGRAL OPERATORS AND SEMICLASSICAL ASYMPTOTICS
}

\author{
A. LAPTEV* \\ Department of Mathematics, Royal Institute of Technology \\ Lindstedtsvägen 25, S-10044 Stockhold, Sweden \\ I. M. SIGAL ${ }^{\dagger}$ \\ Department of Mathematics, University of Toronto \\ 100 St. George Street, Room 4072, M5S 3 G3 Toronto, Canada
}

Received 13 June 1997

Revised 2 April 1999

\begin{abstract}
In this paper we introduce a class of semiclassical Fourier integral operators with global complex phases approximating the fundamental solutions (propagators) for time-dependent Schrödinger equations. Our construction is elementary, it is inspired by the joint work of the first author with Yu. Safarov and D. Vasiliev. We consider several simple but basic examples.
\end{abstract}

Keywords: Fourier integral operator, global phase, Schrödinger equation, fundamental solution, parametrix, semiclassical asymptotics, Morse index, magnetic field.

\section{Introduction}

The notion of Fourier integral operator (FIO) arises in the theory of partial differential equations in two contexts. Firstly, the FIO's realize quantization of classical canonical transformations (Egorov's theorem) (while pseudo-differential operators can be said to arise from quantization of classical observables). More precisely, a transformation of a pseudo-differential operator, $P$, induced by a change of its symbol under a canonical transformation can be realized, in the leading order, by the conjugation, $F P F^{-1}$, by an appropriate FIO $F$. Secondly, the FIO's give a natural construction of approximate fundamental solutions (parametrices) for hyperbolic problems. These two applications are related. Indeed, on the level of symbols (i.e. the level of classical observables) a hyperbolic equation is reduced in the leading order to Hamilton equations. The solution of the latter equations gives a Hamiltonian flow. The latter is a family of canonical transformations (labelled by the time variable). Lifting this family to the level of operators yields a desired approximate solution. This lifting is valid only for sufficiently small times. In general, the construction of a FIO from a flow breaks down at some moment of time due to presence of focal points or caustics. To overcome this problem one either

* Supported by the Swedish NSRC under Grant M-AA/MA 9364-320 and Magnussons fond. † Supported by NSERC under Grant No. NA7901. 
constructs a Maslov canonical operator or a global FIO. Both procedures are rather labor intensive and subtle (see $[12,13,19,21])$.

In this paper we propose a fairly elementary construction of global FIO's giving, in particular, approximate fundamental solutions (or propagators) to the Schrödinger equation

$$
i \alpha \frac{\partial \psi}{\partial t}=H_{\alpha} \psi
$$

where $\alpha$ is a semiclassical parameter and $H_{\alpha}$ is an $\alpha$-differential operator in $\mathbf{R}^{n}$ (see, e.g. $[5,7,10,20,21,26,28]$ ), in terms of semiclassical FIO's. The simplicity of our construction is achieved by using, in our FIO's, complex phases with quadratic imaginary parts. Our approach is inspired by works [17] and [18], where the global parameterization of homogeneous Langrangian distributions was introduced for construction of fundamental solutions of wave equations. Oscillatory integrals with complex phases having quadratic imaginary parts were first considered in $[1,2,3,8,9,16,20,25,27]$ in connection with the problem of propagation of Gaussian wave packets. In another development, FIO's with complex phases were studied in $[22,31]$ in relation to the problem of propagation of singularities for pseudodifferential operators with complex symbols. It seems that none of the works above have isolated the very useful class of FIO's with complex phases having quadratic imaginary parts nor did they use FIO's with complex phases in order to overcome the problem of caustics in deriving quasiclassical or short-wave asymptotics.

Finally we note that in order to avoid the problem of infinite speed of propagation of singularities or oscillations, in the semiclassical context, we localize the construction of an approximate Schrödinger propagator to a part of the phase space, $T^{*} \mathbf{R}^{n}$, in which the momentum (i.e. the cotangent variable) is bounded (cf. [15]).

This paper is organized as follows. In Sec. 2 we introduce our main construction and formulate our main theorem. This theorem is proven in Sec. 3 modulo an important classical statement of independent interest, whose proof is given in Sec. 4 . We use our construction in Sec. 5 in order to obtain semiclassical asymptotics of solutions of Schrödinger Eq. (1.1) with highly oscillating initial conditions. This is a classical problem treated in many texts (see $[6,19,20,21]$ and references therein) which allows a comparison of our method with standard treatments. In Sec. 6 we apply our construction to time-dependent Hamiltonians of a "quadratic type". Our approach allows us to obtain a precise formula for the fundamental solution of the corresponding Schrödinger equation via an oscillatory integral. In the last section we clarify the nature of the phase shift appearing in analysis of the motion of a particle in a singular magnetic potential concentrated at the origin. Some other applications will be presented elsewhere.

In what follows we will be dealing with (vector) functions on $\mathbf{R}^{n}$ and on $\mathbf{R}^{2 n}=$ $T^{*} \mathbf{R}^{n}$. For a function $h(x, \xi)$ from $\mathbf{R}^{2 n}$ to $\mathbf{R}, h_{x}$ denotes the $n$-vector $\left(\frac{\partial h}{\partial x_{1}}, \ldots, \frac{\partial h}{\partial x_{n}}\right)$ (gradient of $h$ in $x)$ and $h_{x \xi}$ the $(n \times n)$-matrix $\left(\frac{\partial^{2} h}{\partial x_{i} \partial \xi_{j}}, i=1, \ldots, n, j=1, \ldots, n\right)$. For a vector function $x(y, \eta)$ from $\mathbf{R}^{2 n}$ to $\mathbf{R}^{n}, x_{\eta}$ stands for the $(n \times n)$ matrix $\left(\frac{\partial x_{j}}{\partial \eta_{i}}, i=1, \ldots, n, j=1, \ldots, n\right)$, etc. $\|\cdot\|$ will denote the norm in $L^{2}\left(\mathbf{R}^{n}\right)$ as 
well as the norm of operators on this space, $x \cdot y=(x, y)$ will denote the real dot product of vectors in $\mathbf{R}^{n}$.

\section{Main Result}

In this section we present the main result of this paper. We begin with some basic definitions. Let $h(t, x, \xi)$ be a real, smooth function on $\mathbf{R}^{1} \times T^{*} \mathbf{R}^{n}$ called a Hamiltonian function. We only assume that there are $m>0$ and $C_{\mu \nu}>0$, such that for every $(t, x) \in \mathbf{R}^{1} \times \mathbf{R}^{n}$

$$
\left|\partial_{x}^{\mu} \partial_{\xi}^{\nu} h(t, x, \xi)\right|<C_{\mu \nu}(1+|\xi|)^{m} .
$$

As usual we denote $D_{x}=-i \frac{\partial}{\partial x}$. Let $H_{\alpha}(t)$ be the $\alpha$-pseudodifferential operator with the symbol $h(t, x, \xi)$, i.e.

$$
\begin{aligned}
H_{\alpha}(t) f(t, x) & =h\left(t, x, \alpha D_{x}\right) f(t, x) \\
& =(2 \pi \alpha)^{-n} \int_{\mathbf{R}^{n}} \int_{\mathbf{R}^{n}} h(t, x, \xi) e^{i(x-y) \xi / \alpha} f(y) d y d \xi,
\end{aligned}
$$

defined first on functions $f \in C_{0}^{\infty}\left(\mathbf{R}^{n}\right)$. We assume that $H_{\alpha}(t)$, for every $t$, has a self-adjoint extention which we continue denoting by the same symbol $H_{\alpha}(t)$. Let $U(t, s)$ be the (Schrödinger) propagator for $H_{\alpha}(t)$, i.e. the family of operators solving the equation

$$
\alpha i \frac{\partial}{\partial t} U(t, s)=H_{\alpha}(t) U(t, s) \text { and } U(s, s)=I
$$

where $I$ stands for the identity operator.

Our task is to construct $\alpha$-FIO's, $U_{N}(t)$, which approximate $U(t, 0)$ within $O\left(\alpha^{N+1-n}\right)$ for any $N \geq 1$. In what follows $\left(x^{t}, \xi^{t}\right)$ denote the solutions of the Hamiltonian equations

$$
\frac{d x^{t}}{d t}=\frac{\partial h}{\partial \xi}, \quad \frac{d \xi^{t}}{d t}=-\frac{\partial h}{\partial x},
$$

subject to the initial conditions

$$
\left.x^{t}\right|_{t=0}=y,\left.\quad \xi^{t}\right|_{t=0}=\eta .
$$

Let $g_{t}$ denote the flow generated by $h$, i.e. $g_{t}(y, \eta)=\left(x^{t}, \xi^{t}\right)$.

Consider the action function $S$ defined as

$$
S(t, y, \eta)=\int_{0}^{t}\left(h_{\xi}\left(s, x^{s}, \xi^{s}\right) \cdot \xi^{s}-h\left(s, x^{s}, \xi^{s}\right)\right) d s .
$$

Definition 2.1. Given $T>0$ and $\Omega \subset T^{*} \mathbf{R}^{n}$, denote by $\phi$ the class of functions (phase functions) $\varphi=\varphi(t, x, y, \eta) \in C^{\infty}\left([0, T) \times \mathbf{R}^{n} \times \Omega\right)$ satisfying the following conditions:

(1) $\varphi\left(t, x^{t}(y, \eta), y, \eta\right)=S(t, y, \eta)$,

(2) $\varphi_{x}\left(t, x^{t}(y, \eta), y, \eta\right)=\xi^{t}(t, y, \eta)$ 
(3) $i^{-1} \varphi_{x x}(t, x, y, \eta) \geq 0$, and is independent of $x$,

(4) $\operatorname{det}\left(\varphi_{x \eta}\left(t, x^{t}(y, \eta), y, \eta\right)\right) \neq 0$ for $(t, y, \eta) \in[0, T) \times \Omega$.

Condition (3) implies that phase functions $\varphi \in \phi$ are polynomials of the second degree with respect to $x$. Expanding $\psi$ satisfying (1)-(3) in $x$ around the point $x^{t}=x^{t}(y, \eta)$, we find the following expression:

$$
\varphi(t, x, y, \eta)=S(t, y, \eta)+\left(x-x^{t}\right) \cdot \xi^{t}+i\left(x-x^{t}\right) \cdot B\left(x-x^{t}\right) / 2,
$$

where $B=B(y, \eta, t)$ is a non-negative definite $n \times n$ matrix $\left(=i^{-1} \varphi_{x x}(t, x, y, \eta)\right)$.

Vice versa, functions of $\psi$ of form (2.7) with $B \geq 0$ and independent of $x$, satisfy conditions (1)-(3). We shall show in Lemma 4.1 below that if $B>0$, then the condition (4) is fulfilled as well and therefore the class $\phi$ is not empty.

Remark 2.1. Instead of the condition (3) we could have considered a more general condition $\Re\left(\left.i^{-1} \varphi_{x x}(t, x, y, \eta)\right|_{x=x^{t}}\right) \geq 0$. This would lead to a wider class of phase function which is useful for analysis on manifolds. However, the class which is introduced in Definition 2.1 is sufficient for our purposes. In particular, for our purposes we can choose $B>0$ and independent of $y, \eta$ and $t$.

The following matrix, appearing in (4), plays an important role in our analysis

$$
Z(t, y, \eta)=\left.\varphi_{x \eta}(t, x, y, \eta)\right|_{x=x^{t}(y, \eta)} .
$$

Note that condition (4) implies that $Z$ is always nonsingular. Differentiating condition (2) with respect to $\eta$, we obtain the following representation of $Z$ :

$$
Z(t, y, \eta)=\xi_{\eta}^{t}(y, \eta)-i x_{\eta}^{t}(y, \eta) B(t, y, \eta)
$$

where we use our convention that $\left(x_{\eta}\right)_{i j}=\frac{\partial x_{j}}{\partial \eta_{i}}$ (see the end of the introduction) and, recalling condition (3), denote

$$
B(t, y, \eta)=i^{-1} \varphi_{x x}(t, x, y, \eta) .
$$

At $t=0$ we obviously have that $\left.Z\right|_{t=0}=I$. To be able to consider the square root of $\operatorname{det} Z$ we introduce the following agreement.

Agreement 2.1. We choose the branch for the function $\operatorname{argdet} Z$ which is continuous in $t$ for $0 \leq t<T$, and which yields zero for $t=0$. This choice allows us to define the value of $(\operatorname{det} Z)^{1 / 2}$ uniquely for all the values of the parameter $t$, $0 \leq t<T$.

We consider a bounded subset $\Omega$ of the phase-space $T^{*} \mathbf{R}^{n}$ and a number $T>0$ satisfying the following condition:

(H) Hamiltonian Eqs. (2.4) and (2.5) have solutions for every $(y, \eta) \in \Omega$ and for $0 \leq t<T$, and the Hamiltonian function $h$ is $C^{\infty}$ on the set $\bigcup_{0 \leq t<T} g_{t}(\Omega)$.

We introduce cut-off functions $\chi, \theta \in C_{0}^{\infty}(\Omega), \chi, \theta \geq 0$ and s.t. $\theta \equiv 1$ on supp $\chi$. With $\chi$ we associate the $\alpha$-pseudodifferential operator $\chi=\chi\left(x, \alpha D_{x}\right)$ :

$$
(\chi f)(x)=(2 \pi \alpha)^{-n} \iint e^{i(x-y) \cdot \eta / \alpha} \chi(y, \eta) f(y) d \eta d y .
$$

Now we are ready to formulate our main result: 
Theorem 2.1. Assume condition $(\mathrm{H})$ to be satisfied. Fix functions $\chi$ and $\theta$ as described above. For any function $\varphi \in \phi$ and integer $N \geq 0$ there is an operator $U_{N}(t)$ such that (i) the Schwartz kernel of $U_{N}(t)$ is of the form

$$
U_{N}(t, x, y)=(2 \pi \alpha)^{-n} \int e^{i \varphi / \alpha} u_{N}(t, y, \eta, \alpha) d \eta,
$$

where $u_{N}=\sum_{k=0}^{N} \alpha^{k} v_{(k)}(\operatorname{det} Z)^{1 / 2}$ with $v_{(k)}$ smooth functions supported in $[0, T) \times$ $\Omega$ and, in particular,

$$
v_{0}(t, y, \eta)=e^{-i \int_{0}^{t} \operatorname{sub} h\left(s, x^{s}, \xi^{s}\right) d s} \theta(y, \eta)
$$

with $\operatorname{sub} h=-\frac{1}{2 i} \operatorname{Tr} h_{x \xi}$, the subprincipal symbol of $h$, and (ii) the operators $U_{N}(t)$ approximate the propagator $U(t, 0)$ on $\Omega$ in the sense that for any $T_{0}<T$

$$
\sup _{0 \leq t \leq T_{0}}\left\|\left(U(t, 0)-U_{N}(t)\right) \chi\right\| \leq C \alpha^{N-2 n},
$$

where $C=C\left(h, \Omega, T_{0}\right)$.

The proof of this theorem is given in the next section.

We leave out an important question of how the constant in (2.14) depends on $h, \Omega$ and $T$, which requires a rather involved analysis. Notice also that (2.12) gives a family of approximate fundamental solutions depending on a choice of the phase function $\varphi$ (or the matrix $B$ in (2.7)).

\section{Proof of Theorem 2.1}

We begin with deriving some simple equalities needed in the proof.

Lemma 3.1. For all $(t, y, \eta) \in[0, T) \times \Omega$ the action function $S$ defined in (2.6), satisfies the following identities:

$$
S_{\eta}=x_{\eta}^{t} \xi^{t}
$$

and

$$
S_{y}=x_{y}^{t} \xi^{t}-\eta
$$

Proof. In the proof below we suppress the superindex $t$. Both sides of (3.1) are equal to zero when $t=0$ since $\left.S\right|_{t=0}=0$ and $\left.x_{\eta} \cdot \xi\right|_{t=0}=y_{\eta} \cdot \eta=0$. Using (2.6), we find

$$
S_{t}=h_{\xi}(t, x, \xi) \cdot \xi-h(t, x, \xi)
$$

Thus in view of $(2.4)$

$$
\begin{aligned}
\frac{\partial}{\partial t} S_{\eta} & =\frac{\partial}{\partial \eta}\left(h_{\xi} \xi-h\right) \\
& =\dot{x} \xi_{\eta}+\dot{x}_{\eta} \xi-x_{\eta} h_{x}-h_{\xi} \xi_{\eta} \\
& =\dot{x}_{\eta} \xi+x_{\eta} \dot{\xi}=\frac{d}{d t}\left(x_{\eta} \xi\right) .
\end{aligned}
$$


This leads to (3.1).

Analogously both sides of (3.2) are equal to zero when $t=0$. Using now (3.3) and (2.4) we have

$$
\begin{aligned}
\frac{\partial}{\partial t} S_{y} & =\frac{\partial}{\partial y}\left(h_{\xi} \cdot \xi-h\right)=\dot{x} \xi_{y}+\dot{x}_{y} \xi-x_{y} h_{x}-h_{\xi} \xi_{y} \\
& =\dot{x}_{y} \xi+x_{y} \dot{\xi}=\frac{d}{d t}\left(x_{y} \xi\right),
\end{aligned}
$$

which completes the proof.

Now we proceed directly to the proof of Theorem 2.1. The proof is straightforward and standard except for one little twist. Since the integration in $\eta$ is over bounded sets, all the estimates occuring below are elementary. We shall separate the proof into three parts.

Step 1. Substitution.

We look for a $\alpha$-FIO $U_{N}(t)$ given by the integral kernel (2.12) which solves the equation

$$
\left(\alpha i \frac{\partial}{\partial t}-H_{\alpha}(t)\right) U_{N}(t)=R_{N}(t),
$$

where $R_{N}$ is a $\alpha$-FIO with the phase $\varphi$ and a symbol $\alpha^{N+2} r_{N}$, where $r_{N}=O(1)$ and $r_{N}(t, \cdot, \cdot) \in C_{0}^{\infty}(\Omega)$. First we compute the integral kernel of the operator $\left(\alpha i \frac{\partial}{\partial t}-H_{\alpha}(t)\right) U_{N}(t)$. Taking into account (2.12), the fact that the phase function $\varphi$ is a quadratic function with respect to $x$ and applying the standard formula for the action of a pseudodifferential operator on an exponential function (see for example [13]), we obtain

$$
\left(\alpha i \frac{\partial}{\partial t}-H_{\alpha}(t)\right) U_{N}(t, x, y)=(2 \pi \alpha)^{-n} \int e^{i \varphi / \alpha}\left[\alpha i \frac{\partial}{\partial t} u_{N}-\left(g+O\left(\alpha^{2}\right)\right) u_{N}\right] d \eta,
$$

where

$$
g=\varphi_{t}+h\left(t, x, \varphi_{x}\right)+\frac{\alpha}{2}\left(\left(B \partial_{\xi}, \partial_{\xi}\right) h\right)\left(t, x, \varphi_{x}\right) .
$$

Since $\varphi$ is a non-degenerated phase function and due to condition (2.1), the r.h.s. of (3.5) can be understood as an oscillatory integral. Using (2.4), (2.6) and (2.7), we find that

$$
\begin{aligned}
\varphi_{t}= & h_{\xi}\left(t, x^{t}, \xi^{t}\right) \cdot \xi^{t}-h\left(t, x^{t}, \xi^{t}\right)-\dot{x}^{t} \cdot \xi^{t}+\left(x-x^{t}\right) \cdot \dot{\xi}^{t} \\
& -i \dot{x}^{t} B\left(x-x^{t}\right)+\frac{i}{2}\left(x-x^{t}\right) \cdot \dot{B}\left(x-x^{t}\right) \\
= & -h\left(t, x^{t}, \xi^{t}\right)-\left(x-x^{t}\right) \cdot h_{x}\left(t, x^{t}, \xi^{t}\right)-i h_{\xi}\left(t, x^{t}, \xi^{t}\right) B\left(x-x^{t}\right) \\
& +\frac{i}{2}\left(x-x^{t}\right) \cdot \dot{B}\left(x-x^{t}\right),
\end{aligned}
$$


where $\dot{B}=\frac{\partial B}{\partial t}$. Applying the Taylor formula, we obtain that the third and the fourth terms on the right-hand side of (3.5) can be written as follows:

$$
\begin{aligned}
h\left(t, x, \varphi_{x}\right)= & h\left(t, x, \xi^{t}+i B\left(x-x^{t}\right)\right) \\
= & h\left(t, x^{t}, \xi^{t}\right)+h_{x}\left(t, x^{t}, \xi^{t}\right) \cdot\left(x-x^{t}\right)+i h_{\xi}\left(t, x^{t}, \xi^{t}\right) \cdot B\left(x-x^{t}\right) \\
& +\frac{1}{2} \sum_{\left|\nu_{1}+\nu_{2}\right|=2}\left(\partial_{x}^{\nu_{1}} \partial_{\xi}^{\nu_{2}} h\right)\left(x-x^{t}\right)^{\nu_{1}}\left(i B\left(x-x^{t}\right)\right)^{\nu_{2}}+O\left(\left|x-x^{t}\right|^{3}\right)
\end{aligned}
$$

and

$$
\frac{\alpha}{2}\left(\left(B \partial_{\xi}, \partial_{\xi}\right) h\right)\left(t, x, \varphi_{x}\right)=\frac{\alpha}{2}\left(\left(B \partial_{\xi}, \partial_{\xi}\right) h\right)\left(t, x^{t}, \xi^{t}\right)+\alpha O\left(\left|x-x^{t}\right|\right) .
$$

Summing up the expressions (3.6)-(3.8), we find that the terms with $\alpha^{0}$ and with $\left(x-x^{t}\right)$ cancel. The rest can be rewritten as

$$
g=g_{1}+\frac{\alpha}{2}\left(\left(B \partial_{\xi}, \partial_{\xi}\right) h\right)\left(t, x^{t}, \xi^{t}\right)+O\left(\left|x-x^{t}\right|^{3}\right)+\alpha O\left(\left|x-x^{t}\right|\right),
$$

where

$$
\begin{aligned}
g_{1}= & \frac{1}{2} \sum_{\left|\nu_{1}+\nu_{2}\right|=2}\left(\partial_{x}^{\nu_{1}} \partial_{\xi}^{\nu_{2}} h\right)\left(x-x^{t}\right)^{\nu_{1}}\left(i B\left(x-x^{t}\right)\right)^{\nu_{2}} \\
& +\frac{i}{2}\left(x-x^{t}\right) \cdot \dot{B}\left(x-x^{t}\right) .
\end{aligned}
$$

Step 2. Integration by parts.

In order to obtain the transport equations we integrate by parts in the integral involving $g_{1}$. Notice that

$$
\frac{\partial}{\partial \eta} e^{i \varphi / \alpha}=\frac{i}{\alpha} e^{i \varphi / \alpha} \varphi_{\eta}=\frac{i}{\alpha} e^{i \varphi / \alpha}\left(S_{\eta}-x_{\eta}^{t} \xi^{t}+\xi_{\eta}^{t}\left(x-x^{t}\right)-i\left(B\left(x-x^{t}\right), x_{\eta}^{t}\right)\right) .
$$

By Lemma 3.1 we find $S_{\eta}-x_{\eta}^{t} \xi^{t}=0$. Therefore taking into account (2.9), we obtain

$$
\left(x-x^{t}\right) e^{i \varphi / \alpha}=-i \alpha Z^{-1} \frac{\partial}{\partial \eta} e^{i \varphi / \alpha} .
$$

Using this equation and integrating twice by parts in order to convert all powers of $\left(x-x^{t}\right)$ into powers of $\alpha$, we obtain

$$
\begin{aligned}
\int e^{i \varphi / \alpha} g_{1} u_{N} d \eta= & \frac{\alpha}{2 i} \int e^{i \varphi / \alpha}\left\{\operatorname { T r } \left[Z ^ { - 1 } \left(h_{x x}+i B h_{x \xi}\right.\right.\right. \\
& \left.\left.\left.+i h_{x \xi} B-B h_{\xi \xi} B+i \dot{B}\right) x_{\eta}^{t}\right] u_{N}+\sum_{|\beta| \leq 2} O(\alpha) \partial^{\beta} u_{N}\right\} d \eta .
\end{aligned}
$$


Note that the leading order here is produced when the $\eta$-derivative is switched from $e^{i \varphi / \alpha}$ onto the remaining (first) power of $x-x^{t}$. Differentiating Hamilton Eq. (2.4) with respect to $\eta$, we obtain

$$
\dot{x}_{\eta}^{t}=x_{\eta}^{t} h_{x \xi}+\xi_{\eta}^{t} h_{\xi \xi}, \quad \dot{\xi}_{\eta}^{t}=-x_{\eta}^{t} h_{x x}-\xi_{\eta}^{t} h_{\xi x} .
$$

(Remember that according to our convention established at the end of the introduction, we have, e.g., $\left(x_{\eta}^{t}\right)_{i j}=\frac{\partial x_{j}^{t}}{\partial \eta_{i}}$ and $\left(h_{x \xi}\right)_{i j}=\frac{\partial^{2} \eta}{\partial x_{i} \partial \xi_{j}}$.) This implies

$$
\begin{aligned}
x_{\eta}^{t}\left(h_{x x}\right. & \left.+i B h_{x \xi}+i h_{x \xi} B-B h_{\xi \xi} B+i \dot{B}\right) \\
= & \left(x_{\eta}^{t} h_{x x}+\xi_{\eta}^{t} h_{x \xi}\right)+i B\left(x_{\eta}^{t} h_{x \xi}+\xi_{\eta}^{t} h_{\xi \xi}\right)+i x_{\eta}^{t} \dot{B} \\
& -\left(\xi_{\eta}^{t}-x_{\eta}^{t} i B\right) h_{x \xi}-\left(\xi_{\eta}^{t}-i B x_{\eta}^{t}\right) i B h_{\xi \xi} \\
= & -\dot{Z}-Z h_{x \xi}-Z i B h_{\xi \xi} .
\end{aligned}
$$

Finally, collecting together (3.5), (3.9), (3.12) and (3.13) and using (3.11) and integration by parts in order to convert the powers of $x-x^{t}$ in the remainder in (3.9) into powers of $\alpha$, we derive

$$
\begin{aligned}
& \left(\alpha i \frac{\partial}{\partial t}-H_{\alpha}(t)\right) U_{N}(t, x, y) \\
& \quad=i(2 \pi)^{-n} \alpha^{-n+1} \int e^{i \varphi / \alpha}\left[\frac{\partial}{\partial t}-\frac{1}{2} \operatorname{Tr}\left(Z^{-1} \dot{Z}+h_{x \xi}\right)+O(\alpha)\right] u_{N} d \eta,
\end{aligned}
$$

where $O(\alpha)$ is a differential operator in $\eta$ with coefficients of order $\alpha$. Consequently, Eq. (3.4) can be rewritten as

$$
i \alpha\left[\frac{\partial}{\partial t}-\frac{1}{2} \operatorname{Tr}\left(Z^{-1} \dot{Z}+h_{x \xi}\right)+O(\alpha)\right] u_{N}=\alpha^{N+2} r_{N} .
$$

Now we write $u_{N}=\sum_{k=0}^{N} \alpha^{k} u_{(k)}$ and equate in Eq. (3.15) the terms containing the same power of the parameter $\alpha$, starting with $\alpha$ and ending with $\alpha^{N+2}$. As a result we obtain the recurrent system of transport equations. The terms with the multiplier $\alpha^{k+1}$ yield the equation

$$
\left.\frac{\partial}{\partial t} u_{(k)}-\frac{1}{2} \operatorname{Tr}\left(Z^{-1} \dot{Z}+h_{x \xi}\right)\right) u_{(k)}=O(1) u_{(k-1)},
$$

where $k=0,1, \ldots, N, u_{(-1)}=0$ and $O(1)$ is a differential operator in $\eta$ with coefficients of order $1\left(O(1)=-\frac{1}{\alpha} O(\alpha)\right.$, where $O(\alpha)$ is the same as in (3.15)). Setting $r_{N}=-O(1) u_{(N)}$, we arrive at Eq. (3.4) for $U_{N}(t)$.

Next, we require that

$$
U_{N}(0)=\theta\left(x, \alpha D_{x}\right)+O\left(\alpha^{N+1}\right),
$$

where $\theta$ is a smooth function supported in $\Omega$ and s.t. $\theta \equiv 1$ on $\operatorname{supp} \chi$ (see the paragraph containing Eq. (2.11)) and $O\left(\alpha^{N+1}\right)$ stands for a bounded operator whose 
norm is bounded by $C \alpha^{N+1}$. This leads to initial conditions for Eq. (3.16), to derivation of which we proceed.

Using Eq. (2.7) for the phase $\varphi$, we obtain

$$
U_{N}(0, x, y)=(2 \pi \alpha)^{-n} \int e^{i(x-y) \cdot \eta / \alpha} e^{-(B(0, y, \eta)(x-y), x-y) / 2 \alpha} u_{N}(0, y, \eta) d \eta .
$$

Expanding the function $\exp [-(B(0, y, \eta)(x-y), x-y)]$ in the Taylor series and integrating by parts, we obtain

$$
U_{N}(0, x, y)=(2 \pi \alpha)^{-n} \sum_{\ell=0}^{\infty} \frac{\alpha^{\ell}}{\ell !} \int e^{i(x-y) \cdot \eta / \alpha} A^{\ell} u_{N} d \eta
$$

where the operator $A$ is defined by

$$
(A g)(\eta)=\sum_{k, \ell=1}^{n} \partial_{\eta_{k} \eta_{\ell}}^{2}\left(b_{k \ell}(0, y, \eta) g(\eta)\right)
$$

with $b_{k \ell}$ being the matrix elements of $B$. Comparing this with (3.17), using the standard definition of a pseudodifferential operator in terms of its symbol, and writting $u_{N}=\sum_{k=0}^{N} \alpha^{k} u_{(k)}$, we obtain

$$
u_{(0)}(0, y, \eta)=\theta(y, \eta)
$$

and, for $k=1,2, \ldots, N$,

$$
u_{(k)}(0, y, \eta)=-\sum_{\ell=1}^{k} \frac{1}{\ell !} A^{\ell} u_{(k-\ell)}(0, y, \eta) .
$$

Linear differential Eq. (3.16) with initial conditions (3.20) and (3.21) can be solved explicitly. In particular,

$$
u_{(0)}(t, y, \eta)=(\operatorname{det} Z(t, y, \eta))^{1 / 2} e^{-i \int_{0}^{t} \operatorname{sub} h\left(s, x^{s}, \xi^{s}\right) d s} \theta(y, \eta),
$$

where, recall $\operatorname{sub} h=-\frac{1}{2 i} \operatorname{Tr} h_{x \xi}$, and similarly for $u_{(k)}, k \geq 1$. The explicit form of the solutions shows that $u_{(k)}(t, \cdot, \cdot) \in C_{0}^{\infty}(\Omega)$ for $k \geq 0$ and $0 \leq t \leq T$.

Remark 3.1. Since every $\left|x-x^{t}\right|^{2}$ is traded for at least the first power of $\alpha$, when integrating by parts, Eq. (3.16) involves no more than $2(N-k+1)$ derivatives of the coefficients $u_{(k)}$.

Step 3. Error estimate (2.14).

Since $u_{N}(t, \cdot, \cdot) \in C_{0}^{\infty}(\Omega)$ and $\Omega$ is compact, we have that $U_{N}(t, x, \cdot) \in C_{0}^{\infty}\left(\mathbf{R}^{n}\right)$. Writing $x$ as $x-x^{t}+x^{t}$ and integrating by parts with a help of (3.11), it is easy to convince oneself that $U_{N}(t, \cdot, y)$ is in the Schwartz class $\mathcal{S}\left(\mathbf{R}^{n}\right)$. The last two properties can be combined into the formula

$$
U_{N}(t, \cdot, \cdot) \in \mathcal{S}\left(\mathbf{R}^{n}, C_{0}^{\infty}(\Omega)\right) .
$$


The definition of $r_{N}$ (see the paragraph after Eq. (3.16)) and an argument similar to one presented above show that

$$
\sup _{0 \leq t \leq T}\left\|R_{N}(t)\right\| \leq C(h, \Omega, T) \alpha^{N+1-n} .
$$

Now integrating Eq. (3.4), we obtain

$$
U_{N}(t)-U(t, 0) U_{N}(0)=\frac{1}{i \alpha} \int_{0}^{t} U(t, s) R_{N}(s) d s .
$$

Multiplying this by the operator $\chi$ from the right and using Eq. (3.24) and the fact that the norm of $U(t, s)$ is uniformly bounded (in fact, is one), we obtain

$$
\left\|\left(U_{N}(t)-U(t, 0) U_{N}(0)\right) \chi\right\| \leq C \alpha^{N-2 n}
$$

with $C=C(h, \Omega, T)$, Finally, due to (3.17) and the fact that $\theta \equiv 1$ on $\operatorname{supp} \chi$,

$$
\left\|U_{N}(0) \chi-\chi\right\| \leq C \alpha^{N-2 n}
$$

and therefore (2.14) follows.

\section{Z-Matrix}

The main result of this section is the following:

Lemma 4.1. If $B>0$, then the r.h.s. of (2.9) defines a non-singular matrixfunction. Consequently, the matrix-function $Z$ introduced in (2.8) is non-degenerate for all values $(t, y, \eta) \in[0, T) \times \Omega$.

Proof. In this proof we write $x^{t}$ as $x(t)$ and similarly for $\xi^{t}$ and often do not display this argument. First we show that

$$
\xi_{\eta} x_{\eta}^{T}=x_{\eta} \xi_{\eta}^{T}
$$

where $\xi_{\eta}^{T}$ is the transpose of the matrix $\xi_{\eta}$. Indeed, $\left.x_{\eta}\right|_{t=0}=0$ and (4.1) is obviously fulfilled for $t=0$. Differentiating equations (2.4) with respect to $\eta$, we find

$$
\frac{d}{d t} x_{\eta}=x_{\eta} h_{x \xi}+\xi_{\eta} h_{\xi \xi}
$$

and

$$
\frac{d}{d t} \xi_{\eta}=-x_{\eta} h_{x x}-\xi_{\eta} h_{\xi x} .
$$

Using these equations it is easy to verify that $\frac{d}{d t}\left(\xi_{\eta} x_{\eta}^{T}\right)=\left(\frac{d}{d t} x_{\eta} \xi_{\eta}^{T}\right)$ and consequently (4.1) is true for all $t$. This proves (4.1) for all $t, 0 \leq t<T$.

Since $B$ is a non-degenerate matrix, it suffices to show that $\operatorname{Ker} Z B^{-1} Z^{*}=\emptyset$. Using (4.1), we obtain

$$
Z B^{-1} Z^{*}=\left(\xi_{\eta}-i x_{\eta} B\right) B^{-1}\left(\xi_{\eta}^{T}+i B x_{\eta}^{T}\right)=\xi_{\eta} B^{-1} \xi_{\eta}^{T}+x_{\eta} B x_{\eta}^{T} .
$$


Let us assume for a moment that $Z B^{-1} Z^{*} c=0$ for some vector $c \in \mathbf{C}^{n}$ and $s$, $0<s<T$. Then, since $B>0,(4.2)$ implies that $\xi_{\eta}^{T}(s) c=x_{\eta}^{T}(s) c=0$. Therefore the system of ordinary differential equations

$$
\begin{aligned}
& \dot{x}_{\eta}^{T} c=h_{\xi x} x_{\eta}^{T} c+h_{\xi \xi} \xi_{\eta}^{T} c \\
& \dot{\xi}_{\eta}^{T} c=-h_{x x} x_{\eta}^{T} c-h_{x \xi} \xi_{\eta}^{T} c
\end{aligned}
$$

for $x_{\eta}^{T}(t) c$ and $\xi_{\eta}^{T}(t) c$, obtained by differentiating (2.4) w.r. to $\eta$, implies that $x_{\eta}^{T}(t) c=0$ and $\xi_{\eta}^{T}(t) c=0$ for all $t \in[0, T]$. This contradicts the relation $\left.\xi_{\eta}\right|_{t=0}=I$.

\section{Quasi-classical Asymptotics}

In this section we study asymptotic behaviour of solutions of the Schrödinger equation

$$
i \alpha \frac{\partial \Psi}{\partial t}=H_{\alpha}(t) \Psi
$$

with initial conditions of the form $\left.\Psi\right|_{t=0}=\Psi_{0 \alpha}=\alpha^{-n / 2} \Psi_{0}\left(\left(x-y_{0}\right) / \alpha\right)$ for some $y_{0} \in$ $\mathbf{R}^{n}$ and $\Psi_{0} \in C_{0}^{\infty}\left(\mathbf{R}^{n}\right)$. Let $\rho(x)$ be a smooth cut-off function, such that $\rho \equiv 1$ on $\operatorname{supp} \Psi_{0 \alpha}$. We write $\Psi_{0 \alpha}$ as $\Psi_{0 \alpha}(x)=\Psi_{0 \alpha}(x) \rho(x)=(2 \pi)^{-n} \rho(x) \int \hat{\Psi}_{0}(\xi) e^{i\left(x-y_{0}\right) \cdot \xi / \alpha}$ $d \xi$, where $\hat{\Psi}_{0}(\xi)$ is the Fourier transform of $\Psi_{0}(x)$. Thus it suffices to consider the initial condition

$$
\left.\Psi\right|_{t=0}=\Psi^{(0)}:=\rho(x) e^{i x \cdot \eta_{0} / \alpha}
$$

for a fixed vector $\eta_{0} \in \mathbf{R}^{n}\left(=T_{y_{0}}^{*} \mathbf{R}^{n}\right)$. For $\alpha \rightarrow 0$, such an initial condition is a fast oscillating function.

The point of taking the initial condition of the form $\alpha^{-n / 2} \Psi_{0}\left(\frac{x-y_{0}}{\alpha}\right)$ is that in this case the average momentum

$$
\int \bar{\Psi}_{0 \alpha}(-i \alpha \nabla) \Psi_{0 \alpha} d^{n} x
$$

is bounded. Another type of the initial condition with bounded average momentum is a direct generalization of $(5.2)$ as

$$
\rho(x) e^{i \nu(x) / \alpha},
$$

where $\nu$ is a smooth function. Although Theorem 5.1 below deals with the initial condition (5.2) a generalization to initial condition (5.3) amounts to the changing just a new notation.

Now observe that the initial value problem (5.1) and (5.2) has the unique solution

$$
\Psi=U_{\alpha}(t, 0) \Psi^{(0)},
$$

where, recall, $U_{\alpha}(t, 0)$ is the propagator from 0 to $t$ associated with (5.1). Due to Theorem 2.1 this propagator is approximated by a global Fourier integral operator 
$U_{N}(t)$ given in Theorem 2.1. Thus we are interested in asymptotic behaviour as $\alpha \rightarrow 0$ of the function

$$
\left(U_{N}(t) \Psi^{(0)}\right)(x)=\int U_{N}(t, x, y) \rho(y) e^{i y \cdot \eta_{0} / \alpha} d y .
$$

The latter is given in Theorem 5.1 below.

Theorem 5.1. Let the conditions of Theorem 2.1 be satisfied and let $U_{N}$ be the approximate evolution operator (2.12). Let a point $\left(t, y_{0}, \eta_{0}\right) \in[0, T) \times \Omega$ be s.t. $\operatorname{det}\left(x_{y}^{t}\left(y_{0}, \eta_{0}\right)\right) \neq 0$. Let $V_{x_{0}^{t}}$ and $V_{y_{0}}$ be neighbourhoods of the points $x_{0}^{t}:=x^{t}\left(y_{0}, \eta_{0}\right)$ and $y_{0}$ s.t. the equation $x=x^{t}\left(y, \eta_{0}\right)$ has a unique solution for $y$ in $V_{y_{0}}$ for any $x \in$ $V_{x_{0}^{t}}$. Then for any smooth function $\rho(y), \operatorname{supp} \rho \subset V_{y_{0}}, \int U_{N}(t, x, y) \rho(y) e^{i y \cdot \eta_{0} / \alpha} d y=$ $0\left(\alpha^{\infty}\right)$ unless $x \in\left\{x^{t}\left(y, \eta_{0}\right) \mid y \in V_{y_{0}}\right\}$. In the latter case we have

$$
\begin{aligned}
& \int U_{N}(t, x, y) \rho(y) e^{i y \cdot \eta_{0} / \alpha} d y \\
& \quad=\rho(\bar{y}) e^{i \bar{y} \cdot \eta_{0} / \alpha} e^{i S\left(t, \bar{y}, \eta_{0}\right) / \alpha} e^{i \operatorname{sub}(t)} e^{i \frac{\pi}{2} m\left(t, \bar{y}, \eta_{0}\right)}\left|\operatorname{det} x_{y}^{t}\left(t, \bar{y}, \eta_{0}\right)\right|^{-1 / 2}+O(\alpha),
\end{aligned}
$$

where $\bar{y}=\bar{y}\left(t, x, \eta_{0}\right)$ is the unique solution of the equation $x^{t}\left(y, \eta_{0}\right)=x, \operatorname{sub}(t)=$ $\int_{0}^{t} \operatorname{sub} h\left(s, x^{s}\left(\bar{y}, \eta_{0}\right), \xi^{s}\left(\bar{y}, y_{0}\right)\right) d s$ and $m(t, y, \eta) \equiv m\left(\gamma^{t}\right)$ is the Morse index of the trajectory $\gamma^{t}=\left\{x^{s}(y, \eta) \mid 0 \leq s \leq t\right\}$.

Proof. We use representation (2.12) for $U_{N}(t, x, y)$ with $B \equiv \frac{1}{i} \varphi_{x x}>0$. Substituting it into the l.h.s. of (5.4), we conclude that the latter is equal to

$$
(2 \pi \alpha)^{-n} \iint e^{i \psi / \alpha} u_{N}(t, y, \eta, \alpha) \rho(y) d \eta d y,
$$

where $\psi(t, x, y, \eta)=\varphi(t, x, y, \eta)+y \cdot \eta_{0}$. We want to apply the stationary phase expansion to this integral. To this end we have to find stationary points of the phase $\psi$ in $\eta$ and $y$ and the Hessian, Hess $\psi$, of $\psi$ at those points. We begin with the former. Using (2.7), (3.1), (2.9) and the fact that $B$ is symmetric, we obtain

$$
\begin{aligned}
\psi_{\eta}=\varphi_{\eta} & =S_{\eta}-x_{\eta}^{t} \xi^{t}+\xi_{\eta}^{t}\left(x-x^{t}\right)-i x_{\eta}^{t} B\left(x-x^{t}\right)+\frac{i}{2}\left(x-x^{t}\right) \cdot B_{\eta}\left(x-x^{t}\right) \\
& =Z\left(x-x^{t}\right)+O\left(\left|x-x^{t}\right|^{2}\right) .
\end{aligned}
$$

Since $\operatorname{det} Z \neq 0$, then in a sufficiently small neigbourhood $V_{x_{0}^{t}}$ of $x_{0}^{t}$ we have

$$
\psi_{\eta}=0 \quad \Rightarrow \quad x=x^{t}(y, \eta) .
$$

Furthermore, (2.7) and (3.2) imply

$$
\begin{aligned}
\varphi_{y} & =S_{y}-x_{y}^{t} \xi^{t}+\xi_{y}^{t}\left(x-x^{t}\right)-i x_{y}^{t} B\left(x-x^{t}\right)+\frac{i}{2}\left(x-x^{t}\right) \cdot B_{y}\left(x-x^{t}\right) \\
& =-\eta+Y\left(x-x^{t}\right)+O\left(\left|x-x^{t}\right|^{2}\right),
\end{aligned}
$$


where

$$
Y(t, y, \eta)=\xi_{y}^{t}(y, \eta)-i x_{y}^{t}(y, \eta) B
$$

Hence

$$
\psi_{y}=\varphi_{y}+\eta_{0}=0 \quad \text { and } \quad(5.7) \quad \Rightarrow \quad \eta=\eta_{0}
$$

Thus on $\operatorname{supp} \rho \times \mathbb{R}^{n}$, the phase $\psi$ has a unique stationary point

$$
\bar{z}=\left(\bar{y}, \eta_{0}\right), \quad \text { where } \bar{y}=\bar{y}\left(t, x, \eta_{0}\right) \text { is the solution to } x^{t}\left(y, \eta_{0}\right)=x,
$$

if $x \in\left\{x^{t}\left(y, \eta_{0}\right) \mid y \in V_{y_{0}}\right\}$ and no stationary points otherwise.

Now we compute the Hessian of the phase function $\psi$ at the stationary point (5.9). First we notice that

$$
\operatorname{Hess} \psi=\operatorname{Hess} \varphi \text {. }
$$

Using expressions (5.7) and (5.9), we compute the matrix Hess $\varphi$ at the point $(t, x, y, \eta)$ s.t. $x^{t}(y, \eta)=x$ :

$$
\operatorname{Hess} \varphi=-\left(\begin{array}{cc}
Y x_{y}^{t} & I+Y x_{\eta}^{t} \\
Z x_{y}^{t} & Z x_{\eta}^{t}
\end{array}\right) .
$$

Note that the relation $\varphi_{\eta y}=\left(\varphi_{y \eta}\right)^{T}$ implies the constraint

$$
I+Y x_{\eta}^{t}=\left(Z x_{y}^{t}\right)^{T} .
$$

Matrix (5.10) can be factorized as follows:

$$
\operatorname{Hess} \varphi=-\left(\begin{array}{cc}
Y x_{y}^{t} & I \\
Z x_{y}^{t} & 0
\end{array}\right)\left(\begin{array}{cc}
I & \left(x_{y}^{t}\right)^{-1} x_{\eta}^{t} \\
0 & I
\end{array}\right),
$$

provided $x^{t}(y, \eta)=x$, which implies

$$
\operatorname{det}(\operatorname{Hess} \varphi)=\operatorname{det} Z \cdot \operatorname{det} x_{y}^{t}, \quad \text { provided } x^{t}(y, \eta)=x .
$$

The next lemma provides the last component needed to assemble the proof.

Lemma 5.1. Let the matrix $x_{y}^{t}\left(y_{0}, \eta_{0}\right)$ be nonsingular. Then, thinking of the sign as +1 or -1 , we have

$$
\operatorname{sign} \operatorname{det} x_{y}^{t}\left(y_{0}, \eta_{0}\right)=(-1)^{m\left(\gamma_{t}\right)}
$$

where $\gamma^{t}$ is the trajectory $\left\{x^{s}\left(y_{0}, \eta_{0}\right) \mid 0 \leq s \leq t\right\}$ and $m\left(\gamma_{t}\right)$, recall, its Morse index.

Proof. By the definition of the Morse index (see [23, 24])

$$
m\left(\gamma^{t}\right)=\sum \text { multiplicity (conjugate point) }
$$

where the sum is taken over all conjugate points along $\gamma^{t}$. Recall that a conjugate point is a point $x^{s_{0}}\left(y_{0}, \eta_{0}\right), 0<s_{0}<t$, at which $\operatorname{det} x_{y}^{s_{0}}\left(y_{0}, \eta_{0}\right)=0$, and the multiplicity of a conjugate point $x^{s_{0}}\left(y_{0}, \eta_{0}\right)$ is the multiplicity of the zero $s=s_{0}$ 
of $\operatorname{det} x_{y}^{s}\left(y_{0}, \eta_{0}\right)$. Since $\left.\operatorname{det} x_{y}^{s}\left(y_{0}, \eta_{0}\right)\right|_{s=0}=1$ and since passing through a conjugate point the sign of the determinant changes by $(-1)^{\text {multiplicity (conjugate point) }}$, we conclude that at the end of the path we have (5.14).

Now we are ready to apply the stationary phase formula (see e.g. [12, Sec. 7.7]) to oscillatory integral (5.5). We use Theorem 2.1 to expand the amplitude $u_{N}$ and Eqs. (5.9) and (5.10) for the critical points and Hessian of the phase. We make two observations. Firstly we note that $(\operatorname{det} Z)^{\frac{1}{2}}$ coming out of $u_{N}$ (see Theorem 2.1) cancels $(\operatorname{det} Z)^{-\frac{1}{2}}$ coming out of $(\operatorname{det} \operatorname{Hess} \varphi)^{-\frac{1}{2}}($ see Eq. (5.13)) (remember that $Z$ is a non-singular matrix). Secondly we see that the second factor on the r.h.s. of (5.13) and Lemma 5.1 leads to

$$
\left(\operatorname{det} x_{y}^{t}\right)^{-\frac{1}{2}}=\left|\operatorname{det} x_{y}^{t}\right|^{-\frac{1}{2}} e^{-i \frac{\pi}{2} m\left(\gamma^{t}\right)},
$$

where, recall, $m\left(\gamma^{t}\right)$ is the Morse index of the trajectory $\gamma^{t}=\left\{x^{s} \mid 0 \leq s \leq t\right\}$. This completes the proof of the theorem.

\section{Quadratic Hamiltonians}

Let $Q(t)$ be a smooth, Hermitian $2 n \times 2 n$ matrix function and $R(t)$, a smooth real $2 n$ vector function. Let $z=(x, \xi) \in \mathbf{R}^{2 n}$ and introduce a quadratic Hamiltonian function by the formula

$$
h(t, z)=\frac{1}{2} z \cdot Q(t) z+R(t) \cdot z .
$$

In this case Hamiltonian system (2.4) with the initial conditions (2.5) can be rewritten as the following system of linear equations

$$
\dot{z}=J(Q(t) \cdot z+R(t)),\left.\quad z\right|_{t=0}=z_{0}=(y, \eta),
$$

where $J=\left(\begin{array}{cc}0 & I \\ -I & 0\end{array}\right)$. Let $M(t)$ be the fundamental matrix of system (6.2), i.e. $M(t)$ solves the initial value problem: $\dot{M}(t)=J Q(t)=M(t)$ and $M(0)=\left(\begin{array}{ll}I & 0 \\ 0 & I\end{array}\right)$. Due to the Liouville-Jacobi formula,

$$
\operatorname{det} M(t)=\exp \left(\int_{0}^{t} \operatorname{Tr} J Q(s) d s\right),
$$

$M(t)$ is a non-degenerate matrix. The solutions $z^{t}$ of $(6.2)$ can be represented in terms of $M(t)$ as

$$
z^{t}=M(t)\left(z^{0}+\int_{0}^{t} M^{-1}(s) J R(s) d s\right) .
$$

Let $m_{i j}(t), i, j=1,2$, be the matrix elements of $M(t)$. According to (2.7) and (2.8) we find

$$
Z(t, y, \eta)=m_{22}(t)-i B(t, y, \eta) m_{12}(t) .
$$

If we use a matrix $B$ which is independent of $(y, \eta)$, then the matrix $Z$ is also independent of $(y, \eta)$ and the first term of the approximation (2.12) to the fundamental solution of Schrödinger Eq. (1.1) gives, in fact, the exact formula for this solution. 
Theorem 6.1. Let $h$ be the Hamilton function defined in (6.1) and let $B=B(t)$ from (2.7) be an arbitrary positive definite matrix independent of $(y, \eta)$ for $t \geq 0$. Then the Schwartz kernel of the Schrödinger Eq. (1.1) is given by the following oscillatory integral

$$
U(t, x, y)=(2 \pi \alpha)^{-n}(\operatorname{det} Z(t))^{1 / 2} \int e^{i \varphi / \alpha} d \eta,
$$

where the phase function $\varphi$ is defined in (2.7) and (2.6).

Proof. The proof can be seen from (3.9). Since the Hamiltonian function is quadratic with respect to $(x, \xi)$, we have that $g=g_{1}$. Moreover, if both $B$ and $Z$ are independent of $(y, \eta)$, then the terms appearing in the sum in (3.12) are equal to 0 .

Notice that (6.4) gives us a family of solutions of the Schrödinger equation depending on the choice of an axiliary matrix $B$. If for example

$$
h(x, \xi)=\frac{1}{2}\left(|\xi|^{2}+|\omega x|^{2}\right)
$$

and $\omega$ is a matrix independent of $t$, then the matrix $M(t)$ in (6.3) can be easiely found to be

$$
M(t)=\left(\begin{array}{cc}
\cos \omega t & \omega^{-1} \sin \omega t \\
-\omega \sin \omega t & \cos \omega t
\end{array}\right) .
$$

If now the matrix $B$ is chosen so that $B=\omega$, then we obtain that $Z$ has a particularly simple form

$$
Z(t, y, \eta)=\cos \omega t-i \sin \omega t=e^{-i t \omega}
$$

and therefore

$$
(\operatorname{det} Z)^{1 / 2}=e^{-i t \operatorname{Tr} \omega / 2} .
$$

Remark 6.1. Notice that $x^{t}(y, \eta)$ and $\xi^{t}(y, \eta)$, defined in (6.3), are linear functions with respect to the initial conditions $(y, \eta)$. Therefore, the phase function in $\varphi$ in (6.4) is quadratic and the integral (6.4) can be computed. In particular, if $h$ is defined by (6.5), then we obtain the well-known Mehler formula for the propagator of the harmonic oscillator.

\section{Schrödinger Operator with Magnetic Potentials}

Now we consider the motion of a particle in a singular magnetic potential concentrated at the origin and having the total flux $\psi_{0}$. Then the corresponding vector potential is $A(x)=\left(A_{1}(x), A_{2}(x)\right)=\psi_{0}\left(-x_{2}, x_{1}\right) /|x|^{2}$. The Schrödinger operator for a particle moving in such a magnetic field is

$$
H_{0 \alpha}=\frac{1}{2}\left(\alpha D_{x}-A(x)\right)^{2},
$$

and the corresponding Hamiltonian function is $h_{0}(x, \xi)=\frac{1}{2}(\xi-A(x))^{2}$. It is a smooth function on $T^{*} \mathbf{R}^{2}$ outside the fiber, $\left\{(x, \xi):(x, \xi)=(0, \xi), \xi \in \mathbf{R}^{2}\right\}$, over 0 . The Hamiltonian equations are 


$$
\left\{\begin{array}{ll}
\dot{x}_{k}^{t}=\xi_{k}^{t}-A_{k}, & \left.x_{k}^{t}\right|_{t=0}=y_{k}, \\
\dot{\xi}_{k}^{t}=\left(\xi^{t}-A\right) \cdot(A)_{x_{k}}, & \left.\xi_{k}^{t}\right|_{t=0}=\eta_{k},
\end{array} \quad k=1,2 .\right.
$$

This system obviously reduces to the system of ordinary differential equations of the second order

$$
\begin{aligned}
\ddot{x}_{k}^{t}= & \sum_{j=1}^{2}\left(\xi_{j}^{t}-A_{j}\right)\left[\left(A_{j}\right)_{x_{k}}-\left(A_{k}\right)_{x_{j}}\right] \\
= & (-1)^{k+1} 2 \pi\left(\xi_{3-k}^{t}-A_{3-k}\left(x^{t}\right)\right) \psi_{0} \delta\left(x^{t}\right), \quad k=1,2, \\
& \left.x^{t}\right|_{t=0}=y,\left.\quad \dot{x}^{t}\right|_{t=0}=\eta-A(y) .
\end{aligned}
$$

If we assume that $x^{t}$ stays away from zero, then Eq. (7.3) can be easily solved

$$
x^{t}(y, \eta)=(\eta-A(y)) t+y, \quad \xi^{t}(y, \eta)=\eta+A\left(x^{t}\right)-A(y) .
$$

From (7.4) we see that $x^{t}(y, \eta)=0$ for some $t$ if and only if $\eta \wedge y:=\eta_{1} y_{2}-\eta_{2} y_{1}=$ $-\psi_{0}$. Therefore we assume that in our construction

$$
\Omega \subset\left\{(y, \eta): \eta \wedge y \neq-\psi_{0}\right\}
$$

Using (2.6) and (7.4), we obtain the expression for the action function

$$
\begin{aligned}
S(t, y, \eta) & =\frac{1}{2}|\eta-A(y)|^{2} t+\int_{0}^{t}(\eta-A(y)) \cdot A\left(x^{s}\right) d s \\
& =\frac{1}{2}|\eta-A(y)|^{2} t-\left(\eta \wedge y+\psi_{0}\right) \psi_{0} \int_{0}^{t}\left|x^{s}\right|^{-2} d s .
\end{aligned}
$$

The integral on the right-hand side multiplying $\psi_{0}$ is a correction term to the free motion due to the magnetic flux through the origin.

Simple calculations show that

$$
\xi_{\eta}^{t}=\left(\begin{array}{cc}
\left(\xi_{1}^{t}\right)_{\eta_{1}} & \left(\xi_{2}^{t}\right)_{\eta_{1}} \\
\left(\xi_{1}^{t}\right)_{\eta_{2}} & \left(\xi_{2}^{t}\right)_{\eta_{2}}
\end{array}\right)=\left(\begin{array}{cc}
1+2 x_{1}^{t} x_{2}^{t}\left|x^{t}\right|^{-4} t \psi_{0} & \left(\left(x_{2}^{t}\right)^{2}-\left(x_{1}^{t}\right)^{2}\right)\left|x^{t}\right|^{-4} t \psi_{0} \\
\left(\left(x_{1}^{t}\right)^{2}-\left(x_{2}^{t}\right)^{2}\right)\left|x^{t}\right|^{-4} t \psi_{0} & 1-2 x_{1}^{t} x_{2}^{t}\left|x^{t}\right|^{-4} t \psi_{0}
\end{array}\right) .
$$

Therefore the determinant,

$$
\operatorname{det} \xi_{\eta}=1-t^{2} \psi_{0}^{2}\left|x^{t}\right|^{-4}
$$

degenerates on the "sphere" $\left|x^{t}\right|=\sqrt{t\left|\psi_{0}\right|}$. At these points of degeneracy a nontrivial matrix $B$ is necessary for our constructions of the corresponding fundamental solution.

Now we consider a particle in $\mathbf{R}^{2}$ moving in the singular magnetic field with the vector potential $A(x)$, given above, and in a quadratic "electric" potential:

$$
H_{\alpha}=\frac{1}{2}\left(\alpha D_{x}-A(x)\right)^{2}+\frac{1}{2}|x|^{2} .
$$


The corresponding Hamiltonian function is

$$
h(x, \xi)=\frac{1}{2}(\xi-A(x))^{2}+\frac{1}{2}|x|^{2} .
$$

The solution of the Hamiltonian Eqs. (2.4) and (2.5) for $y$ and $\eta$, s.t. $x^{t}$ avoids the origin, is

$$
x^{t}=\cos (t) y+\sin (t)(\eta-A(y))
$$

and

$$
\xi^{t}=-\sin (t) y+\cos (t)(\eta-A(y))+A\left(x^{t}\right) .
$$

The action function for this model is equal to

$$
\begin{aligned}
S(t, y, \eta)= & \int_{0}^{t}\left(h_{\xi} \cdot \dot{\xi}^{s}-h\right) d s=\frac{1}{4} \sin (2 t)\left(|\eta-A(y)|^{2}-|y|^{2}\right) \\
& +\frac{1}{2}(\cos (2 t)-1) y \cdot \eta-\psi_{0}\left(\eta \wedge y+\psi_{0}\right) \int_{0}^{t}\left|x^{s}\right|^{2} d s .
\end{aligned}
$$

By analogy with the previous example we see that the integral on the right-hand side of the last expression can be interpreted as a correction term to the motion generated by the harmonic oscillator.

\section{Acknowledgments}

The first author is grateful to L. Kapitanski and Yu. Safarov, while the second author, to V. Ivrii and A. Khovanskii, for many useful discussions.

\section{References}

[1] V. S. Buslaev, "Semi-classical approach for equations with periodic coefficients", Soviet Math. Uspehy 62 (1987) 77-98.

[2] V. M. Babich and V. V. Ulin, "Complex space-time ray method and "quasiphotons"" (in Russian), Zap. Nauchn. Sem. Leningrad. Otdel. Mat. Inst. Steklov. (LOMI) 117 (1981) 5-11.

[3] A. Córdoba and C. Fefferman, "Wave packets and fourier integral operators", Comm. in Partial Diff. Eqs. 3(11) (1978) 979-1005.

[4] M Combescure and D. Robert, "Propagation d'équation de Schrödinger et approximation semi-classique", C. R. Acad. Sci. Paris t. 323, Série I (1996) 871-876.

[5] J. Duistermaat, "Fourier integral operators", in N.Y., Courant Inst. Lect. Notes, 1971.

[6] Yu. V. Egorov and M. A. Shubin (eds.), Partial Diff. Equations I and IV, Encyclopaedia of Mathematical Sciences, Springer-Verlag 1991 and 1993.

[7] V. Guillemin and S. Sternberg, "Geometric asymptotics", Math. Surveys (1977).

[8] G. A. Hagedorn, "Semiclassical quantum mechanics I: The $\hbar \rightarrow 0$ limit for coherent states", Math. Phys. 71 (1980) 77-93.

[9] K. Hepp, "The classical limit for quantum mechanical correlation functions", Commun. Math. Phys. 35 (1974) 265-277.

[10] B. Helffer, "Semiclassical analysis for the Schrödinger operators and applications", Lecture Notes in Mathematics 1336, Springer-Verlag.

[11] B. Helffer and D. Robert, "Propriétés asymptotiques du spectre d'opérateurs pseudodifférentieles sur $\mathbf{R}^{n ",}$ Comm. Partial Diff. Eqs. 7 (1982) 795-882.

[12] L. Hörmander, "Fourier integral operators I", Acta Math. 127 (1971) 79-183. 
[13] L. Hörmander, The Analysis of Linear Partial Differential Operators. I-IV, SpringerVerlag, Berlin, Heidelberg, New York, Tokyo, 1984.

[14] V. Ivrii, Microlocal Analysis and Precise Spectral Asymptotics, Springer, 1998.

[15] V. Ivrii and I. M. Sigal, "Asymptotics of the ground state energy of large Coulomb systems", Ann. Math. 138 (1993) 293-335.

[16] V. V. Kucherenko, "Asymptotic solutions of equations with complex characteristics", Math USSR Sb24 (1976).

[17] A. Laptev and Yu. Safarov, "Global parametrization of Lagrangian manifolds and the Maslov index", preprint, Linköping Univ., 1989.

[18] A. Laptev, Yu. Safarov and D. Vassiliev, "On global representation of Lagrangian manifolds and solutions of hyperbolic equations", Comm. Pure Appl. Math. XLVII (1994) 1411-1456.

[19] V. P. Maslov, Perturbation Theory and Asymptotic Methods, MGU, 1965 (in Russian); French translation: V. P. Maslov, Théorie de Perturbations et Méthodes Asymptotiques, Dunod, Gauthier-Villars, Paris, 1972.

[20] V. P. Maslov, The Complex WKB Method in Nonlinear Equations. I. Linear Theory, Nauka, Moscow, 1977; English translation: Birkhäuser, 1994.

[21] V. P. Maslov and M. V. Fedoryuk, Quasiclassical Approximation for Equations of Quantum Mechanics, Nauka, Moscow, 1976.

[22] A. Melin and J. Sjöstrand, "Fourier integral operators with complex phase functions and parametrix for an interior boundary value problem", Comm. PDE 1 (1975) 313-400.

[23] J. Milnor, Morse Theory Ann. Math. Stud. No. 51, Princeton Univ. Press, 1963.

[24] M. Morse, The Calculus of Variations in the Large AMS Col. Publ. 18, 1934.

[25] T. Paul and A. Uribe, "The semiclassical trace formula and propagation of wave packets", J. Funct. Anal. 132(1) (1995) 192-249.

[26] V. Petkov and G. Popov, "Semi-classical formula and clastering of eigenvalues for Schrödinger operators", Ann. Inst. H. Poincaré, Phys. Theor. 68 (1998) 17-83.

[27] J. Ralston, "Gaussian beams and the propagation of Singularities", MAA Studies in Mathematics 23 (1982).

[28] D. Robert, Autour de l'Approximation Semi-Classique, Birkhäuser, Boston, 1987.

[29] Yu. Safarov and D. Vassiliev, "The asymptotic distribution of eigenvalues of partial differential operators", Transl. of Math. Monographs, ISSN: 0065-9282 155 (1996).

[30] M. Shubin, Pseudodifferential Operators, Springer-Verlag, 1987.

[31] J. Sjöstrand, "Singularités analytiques microlocales", Astérisque 95 (1982). 